\title{
CÂNCER COLORRETAL METASTÁTICO: PAPEL ATUAL DOS ANTICORPOS MONOCLONAIS E A INDIVIDUALIZAÇÃO DE SEU USO
}

\author{
Monoclonal therapy in metastatic colorectal cancer
}

\author{
Fernando Meton de Alencar Camara VIEIRA, Veruska Oliveira DI SENA
}

ABCDDV/638

Vieira FMAC, Di Sena VO. Câncer colorretal metastático: papel atual dos anticorpos monoclonais e a individualização de seu uso. ABCD Arq Bras Cir Dig 2009;22(1):45-9

RESUMO - Introdução - Apesar dos poucos casos de cura no câncer colorretal metastático, a última década foi marcada por avanços na disponibilidade de novos fármacos com mecanismos de ação distintos e aplicabilidade em várias linhas de tratamento. De fato, a sobrevida mediana de pacientes com câncer colorretal metastático praticamente dobrou ao longo dos últimos 10 anos, e parte dessa mudança se deve à introdução dos anticorpos monoclonais, capazes de reconhecer antígenos com importância patogênica em tumores. Objetivo - Apresentar revisão dos resultados obtidos com os anticorpos monoclonais usados em câncer colorretal. Métodos - Revisão de 29 trabalhos publicados e obtidos nas fontes atuais de busca virtual. Foram revisados o papel do Bevacizumabe - anticorpo contra o VEGF; o Cetuximabe e Panitumumabe - anticorpos contra o EGFR e o oncogene K-Ras na resposta ao tratamento. Conclusão - Sugere-se ao final algoritmo de tratamento com anticorpos monoclonais.

DESCRITORES - Cancer. Colo.

\section{INTRODUÇÃO}

O câncer colorretal é a quarta neoplasia mais freqüente entre os homens e a terceira entre as mulheres, acometendo aproximadamente um milhão de pessoas a cada ano no mundo ${ }^{24}$. Apesar da maioria dos pacientes detectar precocemente a neoplasia, com possibilidade de tratamento cirúrgico curativo, cerca de $20 \%$ dos pacientes apresentam doença a distância no momento do diagnóstico ${ }^{21}$. Dentre aqueles submetidos ao tratamento local e adjuvante, $20 \%$ a $40 \%$ apresentarão recidivas sistêmicas durante o acompanhamento clínico ${ }^{5}$. Quando os pacientes apresentam doença a distância, exceto em alguns casos de doença metastática exclusivamente hepática, o tratamento paliativo é indicado ${ }^{26}$ para o aumento da sobrevida e ganho na qualidade de vida. A ressecção curativa de lesões hepáticas pode ser alcançada em pacientes com doença inicialmente ressecável ${ }^{2}$, e em até $16 \%$ dos pacientes com doença inicialmente irresecável, devido às novas estratégias que possibilitam a cirurgia de conversão ${ }^{1}$. A última década foi marcada por avanços na disponibilidade de novos fármacos com mecanismos de ação distintos e com impacto positivo no tratamento seqüencial do câncer colorretal. Com a incorporação dessas novas estratégias, a sobrevida mediana dos pacientes com doença metastática

Trabalho realizado no Serviço de Pesquisa Clínica do Instituto Nacional de Câncer (INCA), Rio de Janeiro, RJ, Brasil.

Endereço para contato: Veruska Oliveira Di Sena. Email: fernandometon@inca.gov.br praticamente dobrou ao longo dos últimos 10 anos, e parte dessa mudança se deve à introdução dos anticorpos monoclonais, capazes de inibir diferentes mecanismos de proliferação tumoral. Os anticorpos monoclonais podem ser quiméricos, humanizados ou humanos. Os anticorpos quiméricos são formados por sequências de aminoácidos de origem humana, com sequências de origem murina nas regiões que se ligam aos epítopos antigênicos. No anticorpos humanizados, grande parte das sequências de aminoácidos é de origem humana, com parcela inferior a $5 \%$ correspondendo a origem murina. Os anticorpos humanos possuem sequências de origem 100\% humana. À medida que se caminha para melhor compreensão do uso destes medicamentos na prática clínica, observa-se a necessidade de identificar biomarcadores relacionados ao prognóstico e predição de benefício.

O objetivo deste artigo foi apresentar revisão dos resultados obtidos com os anticorpos monoclonais usados no tratamento do câncer colorretal.

\section{Bevacizumabe, o anticorpo contra o VEGF}

A angiogênese, fenômeno necessário para o desenvolvimento e a manutenção de tumores, é também alvo para intervenções farmacológicas. A principal molécula pró-angiogênica relacionada ao desenvolvimento tumoral é o fator de crescimento endotelial vascular (vascular endothelial growth factor, VEGF) ${ }^{17}$. Entre as diversas estratégias antiangiogênicas que vêm sendo avaliadas em estudos pré-clínicos e em pacientes com câncer, os melhores resultados foram vistos com o bevacizumabe, o anticorpo monoclonal humanizado contra o VEGF. A utilização do 
bevacizumabe não requer demonstração da expressão de VEGF no espécime tumoral. Da mesma forma, ainda não se conhecem biomarcadores capazes de prever a eficácia desse anticorpo em pacientes com câncer, já que o benefício pelo uso do bevacizumabe não depende das características tumorais avaliadas até o presente momento ${ }^{20,22}$.

$\mathrm{O}$ câncer colorretal foi a primeira patologia em que o bevacizumabe foi aprovado para uso clínico. Essa aprovação teve como base os resultados de um grande estudo randomizado que comparou o tratamento em primeira linha com o regime IFL (irinotecano, fluorouracil em bolus e leucovorin) combinado a placebo ou ao bevacizumabe, na dose de $5 \mathrm{mg} / \mathrm{kg}$ a cada 2 semanas, em 813 pacientes com câncer colorretal avançado ${ }^{19}$. No grupo tratado com IFL e bevacizumabe, a sobrevida mediana foi de 20,3 meses, cerca de cinco meses mais longa que a dos pacientes no grupo tratado apenas com quimioterapia. Além da redução estatisticamente significativa de $34 \%$ do risco de morte, a combinação do bevacizumabe à quimioterapia aumentou a taxa de resposta $(44,8 \%$ versus $34,8 \%-P=0,004)$ e o tempo até a progressão tumoral. As medianas de sobrevida livre de progressão (SLP) foram de 10,6 meses com IFL e bevacizumabe, em comparação com 6,2 meses com IFL e placebo (redução de risco de $46 \%$ - $P<0,001$ ).

Outros estudos randomizados avaliaram o papel da adição do bevacizumabe a regimes quimioterápicos comumente usados no tratamento em primeira linha do câncer colorretal metastático ${ }^{18,23,27}$. Os resultados desses estudos demontraram que a adição do bevacizumabe pode promover aumento da taxa de resposta, do tempo até a progressão e/ou da sobrevida global.

Em segunda linha de tratamento, Giantonio e colaboraradores avaliaram a adição de bevacizumabe com esquema de quimioterapia contendo oxaliplatina (FOLFOX4), em pacientes previamente tratados em primeira linha com esquemas contendo irinotecano em um estudo conhecido com $\mathrm{E} 3200^{11}$. Os autores evidenciaram que a adição de bevacizumabe ao esquema quimioterápico FOLFOX4 alcançou uma duração mediana de sobrevida global de 12,9 meses, quando comparado com o esquema FOLFOX4 isolado, que atingiu 10,8 meses (HR: 0,$75 ; P=0,0011$ ).

Em um estudo observacional, realizado dos Estados Unidos (BRiTE), Grothey e colaboradores avaliaram a reintrodução de bevacizumabe após a progressão em primeira linha. Com essa estratégia, o grupo de pacientes que fizeram uso de bevacizumabe alcançou uma sobrevida mediana de 31,8 meses, quando comparado com o grupo que não fez uso de bevacizumabe em segunda linha, que antigiu uma sobrevida mediana de 19,9 meses ${ }^{13}$. Apesar de ser um estudo observacional, a sobrevida global encontrada sinaliza que a estratégia terapêutica de manter o bevacizumabe em segunda linha poderá ser a nova rotina, após confirmação em estudo randomizados e controlados.

O perfil de toxicidade do bevacizumabe já é bem conhecido e considerado clinicamente manejável. Os principais eventos adversos, muitos deles característicos de medicamentos que interferem com a via de sinalização do VEGF, incluem hipertensão arterial, sangramentos, perfuração gastrointestinal e um discreto aumento da incidência de fenômenos tromboembólicos arteriais. Apesar disso, o bevacizumabe é seguro na grande maioria dos pacientes, sendo infrequente a interrupção do tratamento por eventos adversos graves.

Cetuximabe e panitumumabe, os anticorpos contra o EGFR

O cetuximabe é um anticorpo monoclonal quimérico IgG1 que se liga à porção extra-celular do receptor para o fator de crescimento epidérmico (epidermal growth factor receptor, EGFR). Essa ligação inibe a fosforilação do EGFR e a conseqüente cadeia de eventos bioquímicos que resultariam em estímulo à proliferação celular ${ }^{6}$. O cetuximabe está disponível em diversos países, sendo aprovado no Brasil para o tratamento do câncer de cabeça e pescoço localmente avançado, em combinação com radioterapia ${ }^{8}$. No câncer colorretal metastático refratário a tratamentos anteriores e ao irinotecano, a adição do cetuximabe parece reverter a resistência a esse quimioterápico ${ }^{10}$. Além disso, $\mathrm{o}$ cetuximabe vem sendo avaliado em primeira linha, situação em que sua combinação ao regime FOLFIRI (irinotecano, fluorouracil em infusão contínua e leucovorin) promove modesto aumento da SLP (medianas de 8,9 meses e 8,0 meses, respectivamente, com e sem o anticorpo - $P=0,047$ ) e da taxa de resposta $(46,9 \% \text { versus } 38,7 \%-P=0,005)^{31}$.

O panitumumabe, ainda não disponível no Brasil, é um anticorpo humano que também reconhece o EGFR. Em pacientes com câncer colorretal metastático refratário à quimioterapia, o emprego do panitumumabe promove aumento da SLP, em comparação com o uso exclusivo de tratamento de suporte ${ }^{32}$. Esse benefício, ainda modesto, vem sendo investigado em linhas mais precoces de tratamento. No estudo PACCE (Panitumumab Advanced Colorectal Cancer Evaluation), pretendia-se avaliar a combinação dos dois biológicos, panitumumabe e bevacizumabe, com quimioterapia teve que ser interrompido precocemente, em função do aumento de toxicidade e redução de eficácia decorrentes da associação dos dois anticorpos ${ }^{15,16}$. Aguardam-se as análises definitivas desse estudo, ainda não publicadas.

Tanto o cetuximabe quanto o panitumumabe apresentam perfis de eventos adversos característicos dos inibidores do EGFR, com erupção cutânea acneiforme e diarréia como principais efeitos colaterais. Além disso, ambos podem causar hipomagnesemia ${ }^{12,28}$ e o uso do cetuximabe está associado à ocorrência de reações alérgicas, ocasionalmente graves. Recentemente, demonstrou-se que existe superposição epidemiológica entre a incidência dessas reações e a presença de anticorpos pré-formados, da classe IgE, contra o oligossacarídeo galactose-alfa-1,3-galactose, presente na molécula do cetuximabe ${ }^{9}$. Reações do tipo alérgica ou anafilática também foram reportadas em pacientes que receberam o panitumumabe, muito embora haja ainda menor quantidade de informações a esse respeito ${ }^{11}$. 


\section{O papel do oncogene K-Ras na resposta ao tratamento}

O oncogene K-Ras, localizado no cromossomo 6, codifica uma proteína com importante papel fisiológico e patogênico no câncer colorretal. Em condições normais, em que o gene K-Ras codifica a proteína em seu estado denominado selvagem ou wild type, a ligação de um fator de crescimento ao seu receptor de membrana (p.ex., o EGFR) ativa a proteína Ras (Figura 1). Isso desencadeia uma série de etapas enzimáticas que culminam na ativação de genes importantes na manutenção do fenótipo neoplásico. Na presença de determinadas mutações que podem estar presentes em tumores, em especial nos códons 12 e 13 do gene K-Ras, ocorre ativação da proteína independentemente da presença do fator de crescimento ${ }^{3,13}$. Dessa forma, a presença dessas mutações passou a ser utilizada como biomarcador de resposta ao uso de anticorpos contra EGFR, visto que o bloqueio do receptor passa a não tem efeito terapêutico, quando a proliferação celular está continuamente estimulada, devido a ativação constitutiva da via pela proteína Ras mutada. Além disso, os anticorpos contra o EGFR, um dos principais receptores para fatores de crescimento em tumores, estão contra-indicados nos casos do gene K-Ras mutado, devido aos piores resultados nas respostas ao tratamento. A utilização desses anticorpos está condicionada a análise do gene K-ras e do status selvagem.

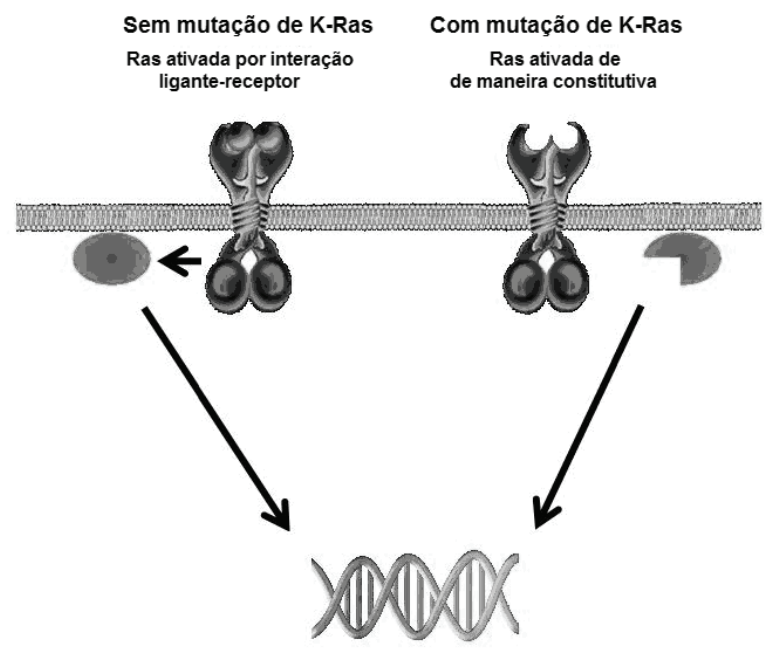

FIGURA 1 - Representação esquemática da conseqüência das mutações do gene K-Ras

De fato, o papel preditivo das mutações de K-Ras foi demonstrado recentemente no caso do panitumumabe, cuja eficácia se limita aos pacientes com tumores sem mutações desse gene ${ }^{4}$. No encontro da American Society of Clinical Oncology de 2008, foi apresentada a análise de subgrupo do estudo CRYSTAL, mencionado anteriormente ${ }^{31}$. Essa análise levou em conta o status mutacional do oncogene K-Ras ${ }^{30}$. Para tanto, foram avaliados os blocos de parafina de 540 dos 1188 pacientes incluídos na análise principal do estudo. As curvas de SLP para esses pacientes foram semelhantes às da população total do estudo, sugerindo que a amostra avaliável para o status mutacional era rep- resentativa dos pacientes incluídos no estudo CRYSTAL. Esses tumores foram submetidos à análise mutacional, que revelou que cerca de dois terços dos casos eram de tumores com gene K-Ras selvagem. A avaliação da SLP nos dois subgrupos de pacientes, mostrada nas Figuras 2 e 3, revelou que o cetuximabe tem seu benefício restrito aos casos em que não há mutação do gene K-Ras.

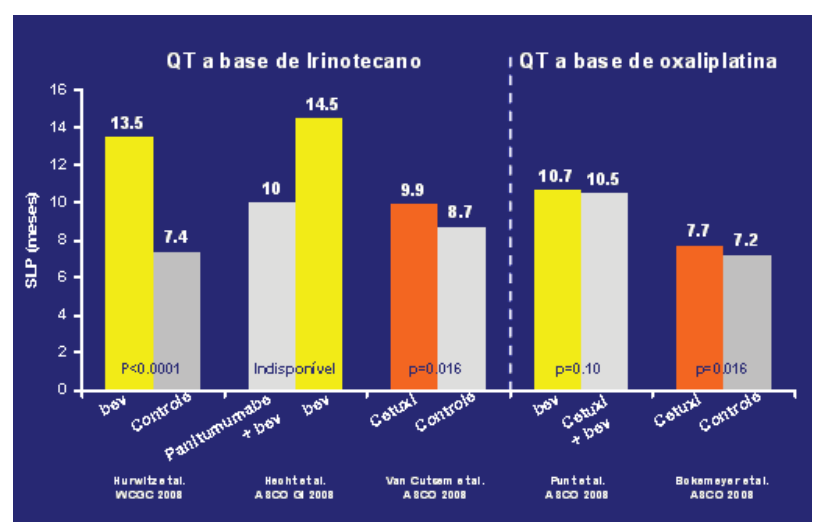

FIGURA 2 - Eficácia das terapias biológicas em K-Ras selvagem: evidência clínica

Esses achados foram confirmados por outro estudo randomizado, de fase II, denominado OPUS, que avaliou a adição do cetuximabe ao tratamento em primeira linha com o regime FOLFOX (fluorouracil, leucovorin e oxaliplatina) ${ }^{7}$. Do total de 337 pacientes incluídos, 233 tiveram seus blocos tumorais analisados para mutações nos códons 12 e 13 do gene K-Ras. Em 42\% dos casos, havia mutação. Os resultados relativos à SLP no estudo OPUS espelham os do estudo CRYSTAL (Figuras 2 e 3 ). Neste caso, entretanto, a diferença desfavorável ao cetuximabe no subgrupo com mutação do gene K-Ras atingiu significância estatística, sugerindo que, nesses casos, a adição do anticorpo pode comprometer o prognóstico.

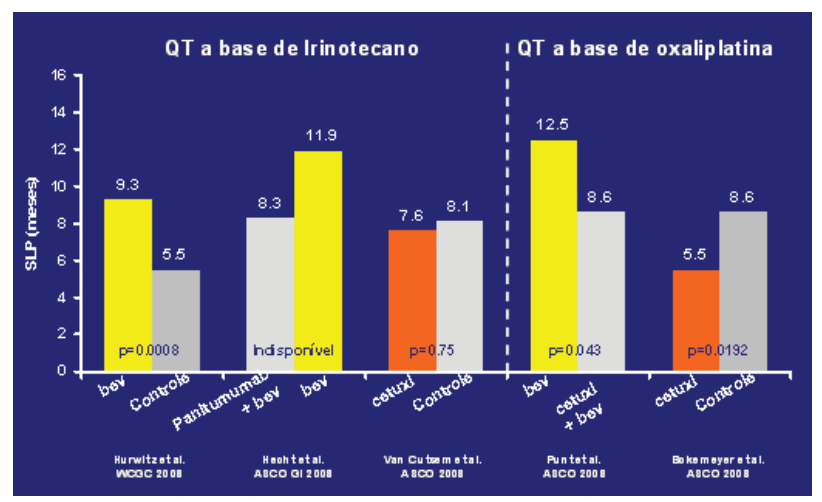

FIGURA 3 - Eficácia das terapias biológicas em K-Ras mutante: evidência clínica

Por fim, a análise de um terceiro estudo randomizado demonstrou o papel preditivo do oncogene K-Ras em pacientes que recebem o cetuximabe. $\mathrm{O}$ estudo holandês CAIRO 2, conduzido por Punt et al. ${ }^{25}$, randomizou pacientes com carcinoma de cólon avançado em primeira linha para 
tratamento com capecitabina, oxaliplatina e bevacizumabe com ou sem cetuximabe. Neste estudo os pacientes cujos tumores apresentam mutação não tiveram benefício com a adição do cetuximabe ao regime XELOX + bevacizumabe (Figuras 2 e 3$)^{25}$. Ao lado do estudo PACCE ${ }^{15}$, este é mais um estudo que desaconselha o uso da combinação de anticorpos monoclonais anti-VEGF e anti-EGFR em primeira linha.

Esses resultados também indicam que o benefício do cetuximabe e do panitumumabe é limitado aos pacientes com tumores sem mutações de K-Ras, sugerindo que o teste para a presença dessas mutações deva ser feito antes da instituição do tratamento com os anticorpos anti-EGFR.

A eficácia do bevacizumabe independe do status do gene $\mathrm{K}-\mathrm{Ras}$, conforme mencionado anteriormente ${ }^{20}$. Embora seja ainda cedo para conclusões definitivas, pode-se encontrar plausibilidade biológica para a independência do efeito do bevacizumabe, com relação às mutações de K-Ras, na própria fisiologia da angiogênese. Muito embora o receptor do tipo 2 para o VEGF, o mais importante em oncologia, também se utilize das vias de sinalização intracelular ativadas pelo EGFR, o receptor do VEGF não ativa necessariamente a proteína Ras, não estando sua sinalização, portanto, aumentada na presença das mutações que conferem atividade constitutiva ao oncogene $\mathrm{K}-\mathrm{Ras}^{29}$. Além disso, deve-se considerar que os receptores para o VEGF são encontrados principalmente no endotélio vascular, e não na célula tumoral, esta última com genoma instável e mais propenso a mutações. O estudo apresentado recentemente por Hurwitz, durante o congresso Mundial de Oncologia do Trato Digestivo em Barcelona, comprovou esta hipótese. Em análise retrospectiva, amostras de tumor primário e de metástases foram obtidas de pacientes que receberam IFL com e sem bevacizumabe no estudo $2107^{19,20}$. Os resultados evidenciaram benefício significativo de SLP independente da mutação no K-Ras. Quando comparadas, as taxas de resposta global são semelhantes em pacientes com K-Ras selvagem usando bevacizumabe ou cetuximabe (Figura 4). Com a análise do K-Ras pode-se definir qual grupo de pacientes se beneficia e qual o apresenta apenas toxicidade. No entanto, ainda não se dispõe de dados de sobrevida global para estes pacientes, sendo este benefício comprovado até o momento, apenas com bevacizumabe.

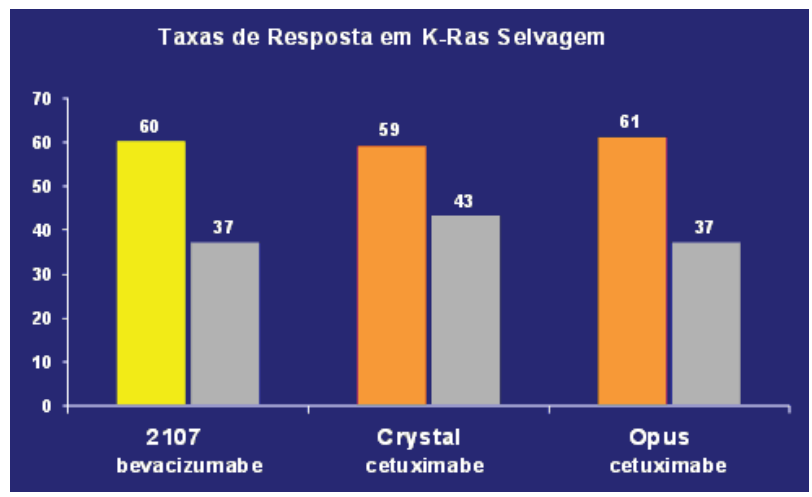

FIGURA 4 - Taxas de resposta em 1a linha de tratamento de câncer colorretal metastático com K-Ras selvagem

\section{Algoritmo atual de tratamento com anticorpos mono- clonais}

O tratamento do câncer colorretal evoluiu muito ao longo dos últimos $10 \operatorname{anos}^{26}$, saindo de uma fase em que a única opção disponível era o fluorouracil. A terapia progressivamente incorporou as fluoropirimidinas orais, tais como a capecitabina, o irinotecano e a oxaliplatina, chegando à fase atual, na qual três anticorpos monoclonais encontram-se disponíveis em diversos países do mundo. Com a disponibilidade de múltiplas intervenções eficazes nas várias linhas de tratamento, cresceu também a complexidade das decisões terapêuticas. Pode-se dizer que, atualmente, não há um algoritmo único, que possa ser usado de maneira universal em câncer colorretal metastático. O que existem, de fato, são opções com níveis de evidência diferentes, conforme a linha que se considera e de acordo com os tratamentos anteriores já utilizados pelos pacientes. Dessa forma, pode-se usar como base para escolha do tratamento a Figura 5, que mostra as principais opções atualmente disponíveis.

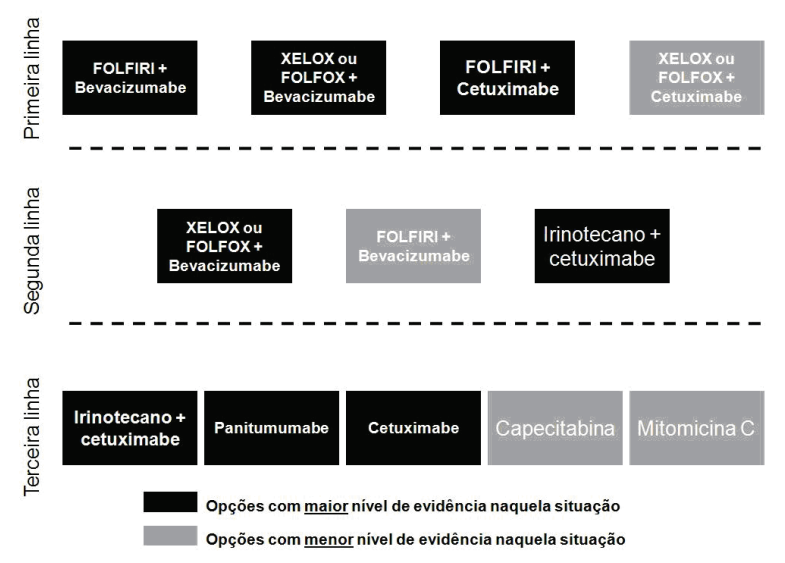

FIGURA 5 - Principais opções atualmente disponíveis nas três primeiras linhas de tratamento em câncer colorretal metastático

A escolha da sequência de tratamento deve ser norteada pelo princípio fundamental de que o emprego de quimioterápicos das três classes disponíveis (fluoropirimidinas, irinotecano e oxaliplatina) está associado a aumento de sobrevida ${ }^{13}$. Além disso, o uso de anticorpos monoclonais deve ser fundamentado em estudos clínicos que espelham a situação em que se encontra o paciente. Por fim, há que se considerar que estamos testemunhando uma fase de grandes mudanças, no âmbito terapêutico, ocasionadas pela maior compreensão da biologia molecular do câncer. Uma das tendências atuais é a individualização do tratamento sistêmico, que vem se tornando possível graças às ferramentas de biologia molecular e às análises estatísticas baseadas em princípios biologicamente plausíveis. Nesse sentido, é de fundamental importância verificar se o paciente apresenta indícios favoráveis ao tratamento com um ou outro agente disponível. É nesse âmbito que devem ser considerados resultados recentes sobre um importante biomarcador no câncer colorretal, o oncogene K-Ras. 


\section{CONCLUSÕES E PERSPECTIVAS FUTURAS}

A introdução do bevacizumabe representou um marco no tratamento do câncer colorretal. O bevacizumabe aumenta significativamente a eficácia da quimioterapia, e seu efeito ainda não foi relacionado à presença de biomarcadores. Os anticorpos anti-EGFR, por sua vez, também são medicamentos valiosos no tratamento do câncer colorretal metastático, muito embora seu benefício pareça estar limitado aos casos em que não há mutações do gene K-Ras. A rigor, o emprego desses agentes, que conferem modesto aumento em termos de resposta objetiva ou SLP em pacientes selecionados, requer a avaliação prévia do espécime tumoral, em busca de mutações que fariam com que o tratamento fosse inativo. É possível que, num futuro próximo, o tratamento de diversos tipos de câncer venha a depender da avaliação de biomarcadores, que farão com que a eficácia terapêutica possa ser otimizada, com redução da toxicidade desnecessária nos casos em que existe evidência molecular de baixo potencial para benefício clínico.

Vieira FMAC, Di Sena VO. Monoclonal therapy in metastatic colorectal cancer. ABCD Arq Bras Cir Dig 2009;22(1):45-9

ABSTRACT - Introduction - Although the metastatic stage of any cancer is difficult to handle, the last decades brought hope with the use of new medications among them the monoclonal antibodies. Method - To update the concepts and use of Bevacizumabe - against VEGF; Cetuximab and Panitumumab - against EGFR and K-Ras oncogene. Conclusion - An algorythm is proposed to better do the treatment.

HEADINGS: Cancer. Colon.

\section{REFERÊNCIAS}

1. Adam R, Wicherts DA, de Haas RJ, Ciacio O, Lévi F, Paule B, Ducreux M, Azoulay D, Bismuth H, Castaing D. Patients with initially unresectable colorectal liver metastases: is there a possibility of cure? J Clin Oncol. 2009 Apr 10;27(11):1829-35. Epub 2009 Mar 9.

2. Adam R. Chemotherapy and surgery: new perspectives on the treatment of unresectable liver metastases. Ann Oncol 2003; 14(Suppl 2):ii13-16.

3. Adjei AA. Blocking oncogenic Ras signaling for cancer therapy. J Natl Cancer Inst 2001; 93:1062-74.

4. Amado RG, Wolf M, Peeters M, et al. Wild-type KRAS is required for panitumumab efficacy in patients with metastatic colorectal cancer. J Clin Oncol 2008; 26:1626-34

5. André T, Boni C, Mounedji-Boudiaf L, et al. Oxaliplatin, fluorouracil, and leucovorin as adjuvant treatment for colon cancer. N Engl J Med 2004; 350:2343-51.

6. Baselga J. The EGFR as a target for anticancer therapy--focus on cetuximab. Eur J Cancer 2001; 37(Suppl 4):S16-22.

7. Bokemeyer C, Bondarenko I, Hartmann JT, et al. KRAS status and efficacy of first-line treatment of patients with metastatic colorectal cancer (mCRC) with FOLFOX with or without cetuximab: The OPUS experience. J Clin Oncol 26: 2008 (May 20 suppl; abstr 4000).

8. Bonner JA, Harari PM, Giralt J, et al. Radiotherapy plus cetuximab for squamouscell carcinoma of the head and neck. N Engl J Med 2006; 354:567-578.

9. Chung CH, Mirakhur B, Chan E, et al. Cetuximab-induced anaphylaxis and IgE specific for galactose-alpha-1,3-galactose. N Engl J Med 2008; 358:1109-17.

10. Cunningham D, Humblet Y, Siena S, et al. Cetuximab monotherapy and cetuximab plus irinotecan in irinotecan-refractory metastatic colorectal cancer. N Engl J Med 2004; 351:337-345.

11. Giantonio BJ, Catalano PJ, Meropol NJ, et al. Bevacizumab in combination with oxaliplatin, fluorouracil, and leucovorin (FOLFOX4) for previously treated metastatic colorectal cancer: results from the Eastern Cooperative Oncology Group Study E3200. J Clin Oncol. 2007 Apr 20;25(12):1539-44.

12. Giusti RM, Shastri K, Pilaro AM, et al. U.S. Food and Drug Administration approval: panitumumab for epidermal growth factor receptor-expressing metastatic colorectal carcinoma with progression following fluoropyrimidine-, oxaliplatin-, and irinotecan-containing chemotherapy regimens. Clin Cancer Res 2008; 14:1296-302.

13. Grothey A, Sargent D. Overall survival of patients with advanced colorectal cancer correlates with availability of fluorouracil, irinotecan, and oxaliplatin regardless of whether doublet or single-agent therapy is used first line. J Clin Oncol 2005; 23:9441-9442.

14. Grothey A, Sugrue MM, Purdie DM, et al. Bevacizumab Beyond First Progression Is Associated With Prolonged Overall Survival in Metastatic Colorectal Cancer: Results From a Large Observational Cohort Study (BRiTE). J Clin Oncol. 2008 Nov 20;26(33):5326-34.

15. Hecht JR, Mitchell E, Chidiac T, et al. An updated analysis of safety and efficacy of oxaliplatin (Ox)/bevacizumab (bev) +/- panitumumab (pmab) for first-line treatment $(\mathrm{tx})$ of metastatic colorectal cancer (mCRC) from a randomized, controlled trial (PACCE). 2008 Gastrointestinal Cancers Symposium, abstract 273.

16. Hecht JR, Mitchell E, Chidiac T, et al. Interim results from PACCE: Irinotecan (Iri)/bevacizumab (bev) \pm panitumumab (pmab) as first-line treatment (tx) for metastatic colorectal cancer (mCRC). 2008 Gastrointestinal Cancers Symposium, abstract 279 .

17. Hicklin DJ, Ellis LM. Role of the vascular endothelial growth factor pathway in tumor growth and angiogenesis. J Clin Oncol 2005; 23:1011-27.
18. Hochster HS, Hart LL, Ramanathan RK, et al. Safety and efficacy of oxaliplatin/ fluoropyrimidine regimens with or without bevacizumab as first-line treatment of metastatic colorectal cancer (mCRC): Final analysis of the TREE-Study. J Clin Oncol 2006; 24:148S (Suppl, abstract 3510).

19. Hurwitz H, Fehrenbacher L, Novotny W, et al. Bevacizumab plus irinotecan, fluorouracil, and leucovorin for metastatic colorectal cancer. N Engl J Med 2004; 350:2335-42.

20. Ince WL, Jubb AM, Holden SN, et al. Association of k-ras, b-raf, and p53 status with the treatment effect of bevacizumab. J Natl Cancer Inst 2005; 97:981-9.

21. Jemal A, Siegel R, Ward E, et al. Cancer statistics, 2008. CA Cancer J Clin 2008; 58:71-96.

22. Jubb AM, Hurwitz HI, Bai W, et al. Impact of vascular endothelial growth factor-A expression, thrombospondin-2 expression, and microvessel density on the treatment effect of bevacizumab in metastatic colorectal cancer. J Clin Oncol 2006; 24:217-27.

23. Kabbinavar FF, Hambleton J, Mass RD, et al. Combined analysis of efficacy: the addition of bevacizumab to fluorouracil/leucovorin improves survival for patients with metastatic colorectal cancer. J Clin Oncol 2005; 23:3706-3712.

24. Parkin DM, Bray F, Ferlay J, Pisani P. Global cancer statistics, 2002. CA Cancer J Clin 2005; 55:74-108.

25. Punt CJ, Tol J, Rodenburg CJ, et al. Randomized phase III study of capecitabine, oxaliplatin, and bevacizumab with or without cetuximab in advanced colorectal cancer (ACC), the CAIRO2 study of the Dutch Colorectal Cancer Group (DCCG). J Clin Oncol 26: 2008 (May 20 suppl; abstr LBA4011).

26. Saad ED, Hoff PM. Chemotherapy of metastatic colorectal cancer. Curr Treat Options Gastroenterol 2005; 8:239-247.

27. Saltz LB, Clarke S, Díaz-Rubio E, et al. Bevacizumab in combination with oxaliplatin-based chemotherapy as first-line therapy in metastatic colorectal cancer: a randomized phase III study. J Clin Oncol 2008; 26:2013-9.

28. Schrag D, Chung KY, Flombaum C, Saltz L. Cetuximab therapy and symptomatic hypomagnesemia. J Natl Cancer Inst 2005; 97:1221-4.

29. Shibuya M. Differential roles of vascular endothelial growth factor receptor-1 and receptor-2 in angiogenesis. J Biochem Mol Biol 2006; 39:469-78. 4

30. Van Cutsem E, Lang I, D'haens G, et al. KRAS status and efficacy in the firstline treatment of patients with metastatic colorectal cancer (mCRC) treated with FOLFIRI with or without cetuximab: The CRYSTAL experience. J Clin Oncol 26: 2008 (May 20 suppl; abstr 2).

31. Van Cutsem E, Nowacki M, Lang I. Randomized phase III study of irinotecan and 5-FU/FA with or without cetuximab in the first-line treatment of patients with metastatic colorectal cancer (mCRC): The CRYSTAL trial. Journal of Clinical Oncology, 2007 ASCO Annual Meeting Proceedings Part I. Vol 25, No. 18S (June 20 Supplement), 2007: 4000.

32. Van Cutsem E, Peeters M, Siena S, et al. Open-label phase III trial of panitumumab plus best supportive care compared with best supportive care alone in patients with chemotherapy-refractory metastatic colorectal cancer. J Clin Oncol 2007; 25:1658-64.

Fonte de financiamento: não há Conflito de interesse: não há Recebido para publicação: 07/08/2008 Aceito para publicação: 16/11/2008 